\title{
Control of Food Intake and Meal Patterns in Monkeys ${ }^{1}$
}

\author{
BARBARA C. HANSEN, KAI-LIN CATHERINE JEN AND LYNNE W. KALNASY \\ Department of Physiology, School of Medicine, University of Michigan, Ann Arbor, MI 48109
}

Received 21 February 1981

\begin{abstract}
HANSEN, B. C., K.-L. C. JEN AND L. W. KALNASY. Control of food intake and meal patterns in monkeys. PHYSIOL. BEHAV. 27(5) 803-810, 1981.-Feeding patterns have been studied in 10 adult male rhesus monkeys (Macaca mulatta) adapted to primate restraint chairs for physiological studies. Using an automated computer-monitored liquid diet feeding system, feeding behavior was studied under 8 hour and 24 hour feeding schedules. Calorie/kg intake was significantly reduced with the 8 hour schedule $(p<0.05)$. Average rate of weight gain and feeding efficiency were also lower with 8 hour feeding, but not significantly different from 24 hour ad lib feeding $(p>0.10)$. Meal size was negatively correlated with meal frequency across monkeys, but not within monkeys. Although monkeys ingested $78 \%$ of their voluntary intake during the light hours, there was no difference in meal size, meal duration, or rate of feeding between light and dark periods. With feeding restricted to $\mathbf{8}$ hours during the light period, meal size was positively correlated with the length of the interval preceding the meal in 4 of 6 monkeys, and meal size was also positively correlated with length of the postmeal interval in 3 of 6 monkeys. In contrast, 24 hour ad lib fed monkeys showed no relationship between premeal interval and subsequent meal size, but a positive correlation between meal size and postmeal interval in 3 of 4 monkeys. We conclude that feeding schedule or deprivation state may alter the relative roles of "hunger" and "satiety" signals in regulating food intake amounts and patterns.
\end{abstract}

Macaca mulatta Feeding pattern Intermeal interval Meal frequency Meal size Meal duration Hunger Satiety

INVESTIGATIONS of the physiology underlying the regulation of feeding behavior depend significantly upon the ability to define and characterize spontaneous patterns of intake under specified nutritional conditions. Studies of feeding regulation have been carried out primarily in rats $[1,2,3,4$, $12,20,21,22]$ and rabbits [5], however, the recent extension of these studies to monkeys [15, 16, 18,25] may provide data important to understanding human feeding behavior. We now present an analysis of feeding patterns and caloric intakes of adult rhesus monkeys, in a model developed specifically to permit sensitive and detailed simultaneous assessment of the multiple behavioral and physiological parameters involved in the regulation of food intake. Results support the use of monkeys in studies of the physiological controls of feeding, and emphasize the importance of within animal comparisons when seeking to demonstrate effects on feeding behavior.

\section{METHOD}

Subjects

The feeding behavior of 10 male rhesus monkeys (Macaca mulatta) was studied under chair-restrained conditions prior to or between experiments requiring chronic intravenous catheterization. Six monkeys were maintained on an 8 hour/day feeding regimen and 4 monkeys had food available 24 hours/day. All monkeys were feral and arrived in the laboratory at least 6 months prior to these studies. The average body weight of these 10 monkeys was $6.8 \mathrm{~kg}$ (4.5 to $10.5 \mathrm{~kg}$ ).

\section{Laboratory Conditions and Diet}

The monkeys were housed in a temperature controlled room $\left(22^{\circ} \mathrm{C}\right)$ with a 12 hour light/dark cycle (lights on at 0700). They were caged and fed Purina Monkey Chow before being switched to a complete liquid diet, Ensure ${ }^{\circledR}$, developed and used for human feeding. The composition of this diet is shown in Table 1 , and contains $1 \mathrm{kcal} / \mathrm{ml}$. All monkeys except 2 received the liquid diet for 1 to 6 months while caged before being placed in the three-tiered primate restraint chairs. The other two were placed on the liquid diet 1 month after chairing. Actual data collection was started after each monkey had been chaired and receiving the liquid diet for a minimum of 2 months. The use of the liquid diet permitted careful quantitative control of the diet composition and quality which, in the present study, were kept constant. Furthermore, unlike for chow feeding, monkeys could not take food to play with, drop, or fill their cheeck pouches when consuming a liquid diet.

'This research was supported by NIH grant AM20493. The authors are grateful to Dr. Harry Kissileff for his critical reading and valuable comments on this paper. Requests for reprints should be sent to Dr. Barbara Hansen, Department of Physiology, School of Medicine, The
University of Michigan, Ann Arbor, MI 48109 . 
TABLE I

COMPOSITION OF ENSURE" (PER LITTER)

\begin{tabular}{|c|c|}
\hline Protein & $37.2 \mathrm{~g}$ \\
\hline Fat & $37.2 \mathrm{~g}$ \\
\hline Carbohydrate & $145.1 \mathrm{~g}$ \\
\hline Calories & $1.06 \mathrm{kcal} / \mathrm{ml}$ \\
\hline Vitamin A & $26431.0 \mathrm{I} . \mathrm{U}$ \\
\hline Vitamin D & $21111.0 \mathrm{I} . \mathrm{U}$ \\
\hline Vitamin E & $321.0 \mathrm{I} . \mathrm{U}$. \\
\hline Vitamin $\mathrm{K}$ & $1.0 \mathrm{mg}$ \\
\hline Vitamin $\mathrm{C}$ & $0.16 \mathrm{~g}$ \\
\hline Folic acid & $0.21 \mathrm{mg}$ \\
\hline Vitamin Bl & $1.6 \mathrm{mg}$ \\
\hline Vitamin $\mathrm{B} 2$ & $1.8 \mathrm{mg}$ \\
\hline Vitamin B6 & $2.1 \mathrm{mg}$ \\
\hline Vitamin B12 & $6.3 \mu \mathrm{g}$ \\
\hline Niacin & $21.0 \mathrm{mg}$ \\
\hline Choline & $0.53 \mathrm{~g}$ \\
\hline Biotin & $0.16 \mathrm{mg}$ \\
\hline Panotothenic Acid & $5.3 \mathrm{mg}$ \\
\hline Sodium & $0.74 \mathrm{~g}$ \\
\hline Potassium & $1.3 \mathrm{~g}$ \\
\hline Chloride & $1.1 \mathrm{~g}$ \\
\hline Calcium & $0.53 \mathrm{~g}$ \\
\hline Phorphorus & $0.53 \mathrm{~g}$ \\
\hline Magnesium & $0.21 \mathrm{~g}$ \\
\hline Iodine & $0.079 \mathrm{mg}$ \\
\hline Manganese & $2.1 \mathrm{mg}$ \\
\hline Copper & $1.1 \mathrm{mg}$ \\
\hline Zinc & $16 \mathrm{mg}$ \\
\hline Iron & $9.5 \mathrm{mg}$ \\
\hline
\end{tabular}

\section{Feeding System}

The feeders used in these studies were designed to provide each monkey with free access to food, while simultaneously permitting recording of detailed patterns of ingestion. The feeding system was an adaptation of our previously described liquid diet feeder [24], and consisted of a doublelumened mouthpiece, a liquid diet reservoir, and a vacuum switch-triggered pump. The mouthpiece was positioned on the top tier of the chair, so the monkey's mouth could easily reach it. One lumen of the mouthpiece connected to tubing which led to a vacuum switch. Upon closure, the switch triggered the pump to deliver $1 \mathrm{ml}$ of liquid diet through separate tubing to the second lumen of the mouthpiece. Thus, each suck by the monkey resulted in the instantaneous delivery of liquid diet into its mouth and no spillage or loss of diet was possible. The liquid diet pumps and their diet reservoirs were located in the room adjacent to the monkeys, and tubes passed through the walls to reach the mouthpieces. Thus the pumps and diet reservoirs could be monitored and refilled without disturbing the monkeys. Diet was delivered to the monkey at room temperature.

\section{Procedure}

For those monkeys on the 8 hour feeding schedule, feeders were set up at 0800 . Fresh water was supplied at the same time and was available for 24 hours a day. The feeder mouthpieces were removed from the chair after 8 hours of feeding. For the monkeys having food access for 24 hour per day, each morning between 0730 and 0800 , the feeding tubes were removed for cleaning and clean lubes were re placed. Water was continuously available 10 all monkeys. The Ensure "ieft in the bottle was carefully measured and recorded as a double check on the computer-monitored feeding records. The monkeys were weighed 3 times a week before clean feeders were presented to them

\section{Data Analysis}

Output from each pump was continuously recorded directly on a mini-computer and subsequently transferred to a CDC 6400 computer for further statistical analysis.

Means of meal frequency, meal size, meal duration and intermeal intervals were calculated for each monkey and group means were then derived by averaging the means for each monkey. Student's $t$-test was performed to compare the 2 groups. Correlations between feeding parameters were also obtained for each monkey.

\section{RESULTS}

The chair-restrained monkey, permitted to ingest only a complete liquid diet, provided an excellent model for the assessment of the determinants of feeding behavior. Animals ingested caloric amounts similar to caged monkeys and in quantities sufficient to provide for continued normal growth and excellent health. The chair restraint appeared to have minimal effect on energy requirements when compared to individually caged animals, and permitted continuous physiological monitoring without complications $[7,17]$. We have assessed the weight changes, caloric intakes and feeding patterns of these monkeys.

\section{Daily Caloric Intakes and Body Weights}

Six monkeys were studied using an 8-hour per day feeding schedule for a total of 771 meals and 1378 -hour periods. Four additional monkeys were studied with food available 24 hours per day for a total of 58 days and 532 meals. Data for each individual monkey and the means across animals are presented in Table 2 . Total daily intake (mean $\pm S E$ ) and intake per $\mathrm{kg}$ of body weight were significantly higher for monkeys on 24 hour feeding schedules than for monkeys fed only 8 hours a day $(671 \pm 71.2 \mathrm{kcal}$, vs $482 \pm 47.6 \mathrm{kcal}$ or 82.9 $\mathrm{kcal} / \mathrm{kg}$ vs $66.7 \mathrm{kcal} / \mathrm{kg}, t=2.30, p<0.05$ ).

Monkeys feeding $8 \mathrm{hr} /$ day showed lower rates of weight gain than those on 24 hour ad lib feeding; however, differences failed to reach significance $(0.098 \pm 0.01 \mathrm{~kg} /$ week for the 8 hour group and $0.189 \pm 0.01 \mathrm{~kg} /$ week for the 24 hour feeding group, $p>0.10$ ). Feeding efficiency was also generally lower under 8 hours/day feeding conditions, but again the difference between 24 hour feeding was not significant $\left(0.23 \pm 0.04 \times 10^{-4} \mathrm{~kg}\right.$ weight gain $/ \mathrm{kcal}$ for the 8 hour group and $0.36 \pm 0.09 \times 10^{-4} \mathrm{~kg}$ weight gain/kcal for the 24 hour group.) Monkeys in both groups showed varied rates of weight gain. Even within the same monkey, rate of weight gain varied (maximum range for one monkey from -0.0060 $\mathrm{kg} /$ week to $0.25 \mathrm{~kg} /$ week), as we have previously described [7].

\section{Determination of Meal Definition}

In order to determine the best criteria for defining a meal, data collected from the 24 hour feeding group were analyzed using selected meal definitions which permitted comparison 
TABLE 2

FEEDING PARAMETERS (MEAN \pm SD)

\begin{tabular}{|c|c|c|c|c|c|}
\hline Monkey & $\begin{array}{l}\text { Mean Total } \\
\text { Daily Intake } \\
\text { (kcal) }\end{array}$ & $\begin{array}{c}\text { Mean Meal } \\
\text { Frequency } \\
\text { (per day) }\end{array}$ & $\begin{array}{c}\text { Mean Meal } \\
\text { Size (MS, } \\
\text { kcal) }\end{array}$ & $\begin{array}{c}\text { Mean Meal } \\
\text { Duration } \\
\text { (MD, min) }\end{array}$ & $\begin{array}{c}\text { Mean } \\
\text { Intermeal } \\
\text { Interval } \\
\text { (IMI, min) }\end{array}$ \\
\hline \multicolumn{6}{|c|}{8 hour/day Feeding Group } \\
\hline S-3 & $428 \pm 125$ & $10.3 \pm 3.6$ & $41 \pm 34$ & $2.2 \pm 1.2$ & $44 \pm 38$ \\
\hline $\mathrm{U}-3$ & $365 \pm 134$ & $2.4 \pm 0.6$ & $155 \pm 84$ & $10.1 \pm 4.8$ & $208 \pm 163$ \\
\hline W-3 & $449 \pm 146$ & $5.3 \pm 1.9$ & $85 \pm 73$ & $5.4 \pm 4.3$ & $79 \pm 73$ \\
\hline$X-3$ & $440 \pm 121$ & $3.9 \pm 1.0$ & $113 \pm 45$ & $8.2 \pm 2.7$ & $107 \pm 58$ \\
\hline $\mathbf{F}-4$ & $700 \pm 126$ & $8.4 \pm 2.1$ & $82 \pm 85$ & $3.4 \pm 3.2$ & $54 \pm 35$ \\
\hline $\mathrm{G}-4$ & $512 \pm 120$ & $7.3 \pm 4.7$ & $70 \pm 75$ & $6.9 \pm 6.4$ & $52 \pm 66$ \\
\hline Mean $\pm S E$ & $482 \pm 47.6$ & $6.3 \pm 1.2$ & $91 \pm 16$ & $6.0 \pm 1.2$ & $91 \pm 25.3$ \\
\hline \multicolumn{6}{|c|}{24 hour/day Feeding Group } \\
\hline C-4 & $620 \pm 227$ & $5.4 \pm 1.8$ & $113 \pm 87$ & $7.1 \pm 4.6$ & $235 \pm 186$ \\
\hline D-4 & $557 \pm 67$ & $15.6 \pm 4.8$ & $35 \pm 29$ & $3.4 \pm 2.5$ & $86 \pm 102$ \\
\hline E-4 & $626 \pm 175$ & $8.4 \pm 2.6$ & $74 \pm 56$ & $6.3 \pm 4.4$ & $148 \pm 128$ \\
\hline $\mathrm{H}-4$ & $879 \pm 141$ & $8.1 \pm 1.5$ & $109 \pm 55$ & $3.1 \pm 1.4$ & $164 \pm 240$ \\
\hline Mean $\pm S E$ & $671 \pm 71.2$ & $9.4 \pm 2.2$ & $83 \pm 18$ & $5.0 \pm 1.0$ & $158 \pm 31$ \\
\hline
\end{tabular}

of the effects of several different minimal intermeal intervals (IMI's) and minimal meal sizes (MS's). Minimum IMI's tested were $2 \mathrm{~min}, 10 \mathrm{~min}$, and $30 \mathrm{~min}$. The minimum MS's tested for use in defining a meal or bout were $2,10,20$, and $40 \mathrm{ml}$. Table 3 summarizes the most useful permutations. The smallest meal size considered $(2 \mathrm{ml})$ was combined with the smallest IMI tested $(2 \mathrm{~min})$ to produce the minimal definition of a meal. Use of this definition resulted in the exclusion of less than $1 \%$ of daily total intake. Changing this meal definition from $2 \mathrm{~min} / 2 \mathrm{ml}$ to $10 \mathrm{~min} / 10 \mathrm{ml}$ significantly changed the description of feeding in 2 of the 4 monkeys. The mean changes for the four monkeys with change in meal definition from $2 / 2$ to $10 / 10$ included a decrease in number of meals per day by $54 \%$, and increases in MS by $22 \%$, meal length by $28 \%$, and IMI by $23 \%$. Further increases in the parameters used to define a meal (i.e., $10 \mathrm{~min} / 20 \mathrm{ml}$ ) did not alter the description of feeding patterns in 3 of the 4 monkeys.

Figure 1 illustrates the distribution of intermeal intervals obtained using the various meal definitions. The $2 \mathrm{ml} / 2 \mathrm{~min}$ definition provided for the inclusion of a large number of small IMI's which were missed by the definitions which required $10 \mathrm{~min}$ or more between feedings. Similarly, Fig. 2 provides the distribution of meal sizes for each meal definition and indicates that a significant number of meals ranged in size between $2 \mathrm{kcal}$ and $10 \mathrm{kcal}$ and would have been missed by selection of the larger volume as the minimal MS criterion. This is further illustrated by comparing typical 24 hour cumulative records selected from 2 monkeys, (Fig. 3), one whose pattern would be significantly changed by increasing meal definition from $2 / 2$ to $10 / 10$ and another for whom such a change in definition would have minimal effect.

TABLE 3

EFFECTS OF VARYING MEAL DEFINITION $($ MEAN \pm SE)*

\begin{tabular}{|c|c|c|c|c|c|c|c|c|c|}
\hline \multirow{2}{*}{$\begin{array}{l}\text { Meal } \\
\text { Definition }\end{array}$} & \multirow{2}{*}{$\begin{array}{l}\text { \% Daily } \\
\text { Intake } \\
\text { Eliminated }\end{array}$} & \multicolumn{2}{|c|}{$\begin{array}{l}\text { Mean Meal } \\
\text { Frequency }\end{array}$} & \multicolumn{2}{|c|}{$\begin{array}{l}\text { Mean Meal } \\
\text { Size (kcal) }\end{array}$} & \multicolumn{2}{|c|}{$\begin{array}{c}\text { Mean Meal } \\
\text { Duration (min) }\end{array}$} & \multicolumn{2}{|c|}{ IMI (min) } \\
\hline & & Frequency & Change ${ }^{\dagger}$ & Size & Change & Duration & Change & Duration & $\begin{array}{c}\% \\
\text { Change }\end{array}$ \\
\hline $2 \mathrm{~min} / 2 \mathrm{ml}$ & $1.0 \%$ & $9.30 \pm 4.28$ & & $82.9 \pm 36.20$ & & $5.0 \pm 2.02$ & & $158.0 \pm 61.22$ & \\
\hline $10 \mathrm{~min} / 10 \mathrm{ml}$ & $2.2 \%$ & $4.32 \pm 1.46$ & $-54 \%$ & $101.1 \pm 40.18$ & $+22 \%$ & $6.4 \pm 2.62$ & $+28 \%$ & $195.1 \pm 72.33$ & $+23 \%$ \\
\hline $10 \mathrm{~min} / 20 \mathrm{ml}$ & $5.3 \%$ & $3.85 \pm 1.01$ & $-59 \%$ & $107.8 \pm 40.87$ & $+30 \%$ & $6.7 \pm 2.81$ & $+34 \%$ & $215.6 \pm 68.46$ & $+36 \%$ \\
\hline $10 \mathrm{~min} / 40 \mathrm{ml}$ & $10.5 \%$ & $3.39 \pm 0.62$ & $-64 \%$ & $113.0 \pm 37.16$ & $+36 \%$ & $7.2 \pm 2.76$ & $+44 \%$ & $239.8 \pm 58.42$ & $+52 \%$ \\
\hline $30 \mathrm{~min} / 10 \mathrm{ml}$ & $2.2 \%$ & $4.00 \pm 1.16$ & $-57 \%$ & $106.5 \pm 39.23$ & $+28 \%$ & $8.2 \pm 2.99$ & $+64 \%$ & $205.0 \pm 73.12$ & $+30 \%$ \\
\hline $30 \mathrm{~min} / 20 \mathrm{ml}$ & $5.3 \%$ & $3.67 \pm 0.82$ & $-61 \%$ & $111.2 \pm 37.67$ & $+34 \%$ & $8.3 \pm 3.00$ & $+66 \%$ & $220.6 \pm 64.42$ & $+40 \%$ \\
\hline
\end{tabular}

*Data derived from 4 monkeys allowed to feed 24 hours per day for a total of 58 days. Averages of the means of these 4 monkeys are presented.

†\% change relative to results using the $2 \mathrm{~min} / 2 \mathrm{ml}$ definition. 


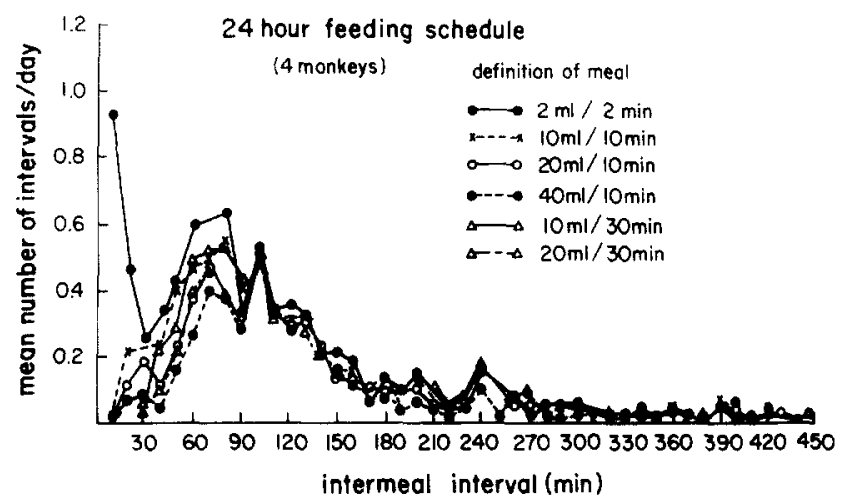

FIG. 1. Distribution of intermeal intervals using 6 definitions of a "meal." Data were derived from 4 monkeys with food available 24 hours/day.

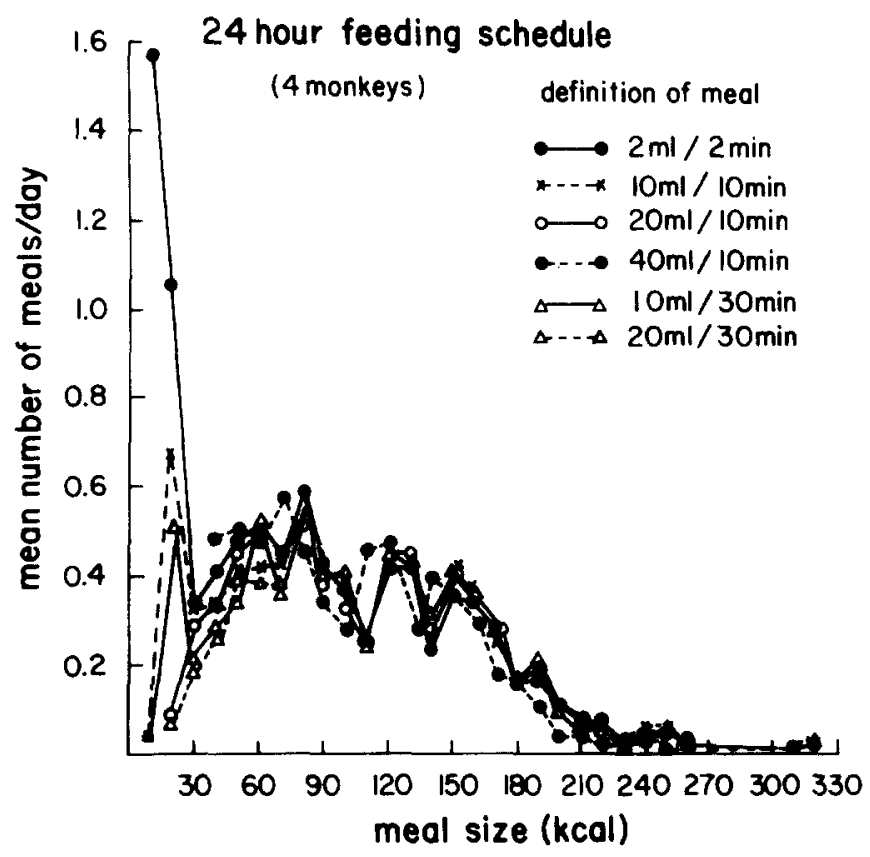

FIG. 2. Distribution of meal sizes using 6 definitions of a "meal." Per Fig. 1, data were from 4 monkeys feeding 24 hours/day.

Although both monkeys had almost the same daily intake, it was achieved via different means. Each monkey had its own relatively consistent pattern of feeding.

Because we wished to use a meal definition which would be sensitive to future experiments in which altered feeding patterns under external or internal manipulations were to be assessed, we selected the $2 / 2$ definition. It was important that patterns of feeding as clearly different as the two shown in Fig. 3 be readily differentiated statistically as we anticipated possible shifts between these feeding patterns under various experimental protocols. Furthermore, the definition of $2 / 2$ covered almost all of the daily intake of all monkeys. Only $1 \%$ of total intake was lost by this definition.

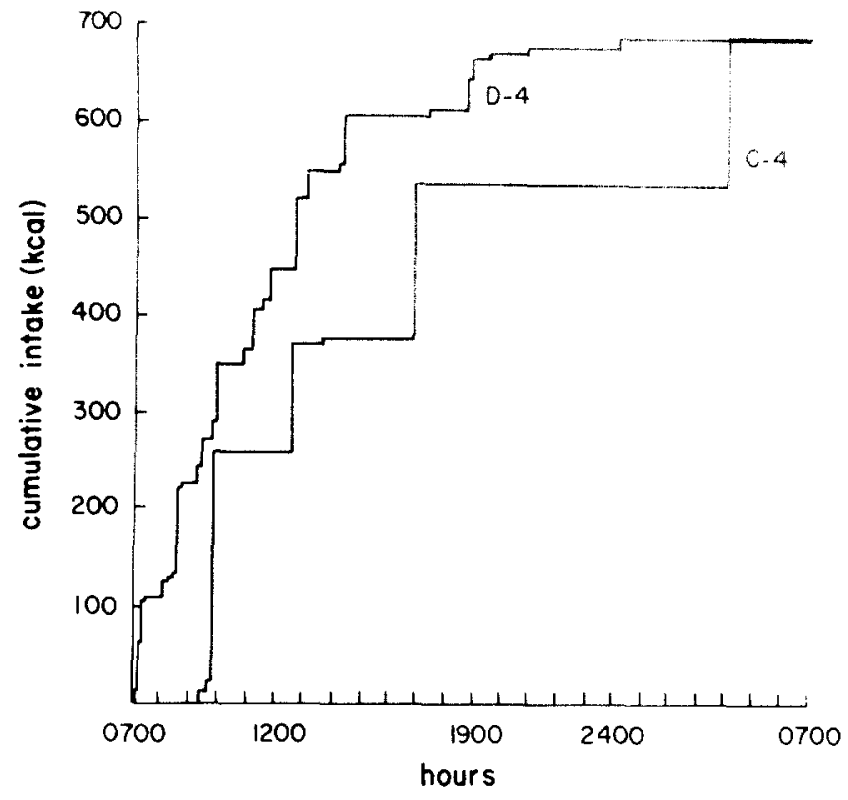

FIG. 3. Typical cumulative intake records for 2 monkeys. As shown, pattern of voluntary intake can differ markedly between monkeys.

Meal frequency was very consistent within monkeys, however, across monkeys, a significant negative correlation between meal size and meal frequency was found $(r=-.863$, $p<0.005$ ), as depicted in Fig. 4. Mean meal size vs mean meal frequency is plotted for each monkey. The difference in feeding patterns between monkeys is clearly shown. Total daily intake $/ \mathrm{kg}$ body weight as noted above, varied widely across monkeys, and was not consistently related to either meal size or meal frequency.

\section{Feeding Patterns During Eight Hour Feeding Schedule}

Monkeys in this group took a mean of 6.3 meals each day (Table 2) and the average number of meals per day varied widely between monkeys (range 2.4 meals to 10.3 meals). There was, however, a consistency within monkey across days. The mean MS for this group of monkeys was $91 \pm 16$ $\mathrm{kcal}$ (range of means for individual monkeys: 41 to $155 \mathrm{kcal}$ ). The frequency distribution for meal size for all monkeys showed that $31 \%$ of the meals were less than $30 \mathrm{kcal}$ while $12 \%$ were greater than $180 \mathrm{kcal}$ (Fig. 5).

We noted that the first and the last meal taken in the $8 \mathrm{hr}$ feeding group appeared to be larger. Therefore, the daily meals were grouped into first meal, last meal and the intervening meals (Table 4). After a 16 hour fast, monkeys in the 8-hour/day feeding schedule usually took a significantly larger first meal. The mean MS of this first meal was 160 kcal, which was significantly larger than the intervening meals (74 kcal, $t=3.24, p<0.01$ ) or the last meal (98 kcal, $t=2.18, p<0.05$ ). In this group, the last meal was determined not by the monkeys, but by the set time of 1600 when food was removed. Monkeys did, however, learn to anticipate a long period without food available; they took a slightly, but not significantly larger last meal $(98 \mathrm{kcal})$ in the 1500 to 1600 hour period compared to the intervening meals $(74 \mathrm{kcal})$. 


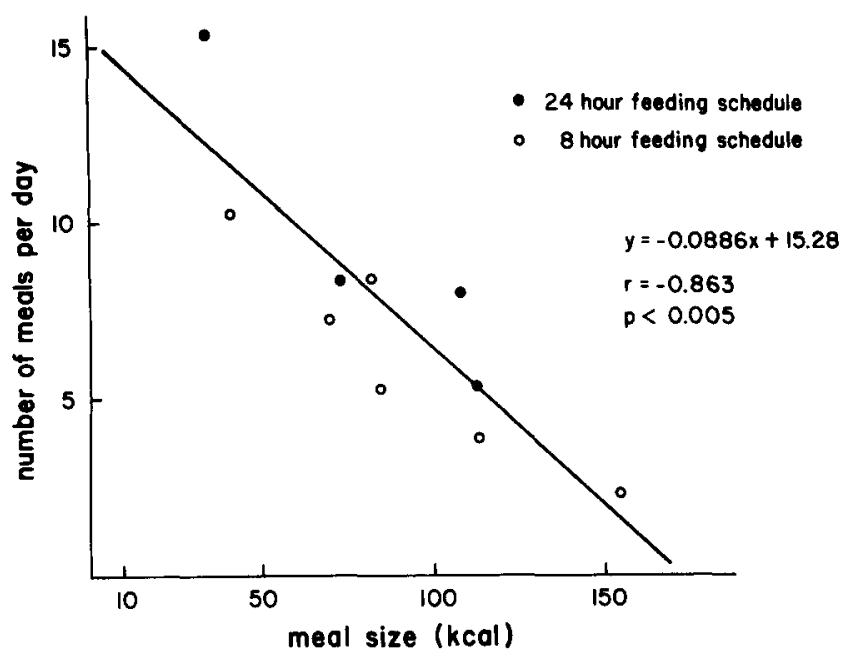

FIG. 4. Mean meal size vs mean meal frequency for 9 monkeys. Two feeding schedules were used ( 24 hours or 8 hours/day).

TABLE 4

MEAN SIZES (kcal) OF FIRST MEAL, INTERVENING MEALS, AND LAST MEAL FOR THE 2 FEEDING GROUPS

\begin{tabular}{lcc}
\hline First Meal & $\begin{array}{c}\text { Intervening } \\
\text { Meals }\end{array}$ & Last Meal \\
\hline
\end{tabular}

8 hour Feeding Group

$\begin{array}{lcrr}\text { R-3 } & 180.86 & 110.00 & 138.57 \\ \text { S-3 } & 75.78 & 39.88 & 50.78 \\ \text { W-3 } & 175.82 & 63.41 & 87.86 \\ \text { X-3 } & 154.06 & 96.91 & 107.78 \\ \text { F-4 } & 251.33 & 58.91 & 68.25 \\ \text { G-4 } & 118.26 & 73.08 & 133.74 \\ \text { Mean } & 159.52^{*+} & 73.70 & 97.82 \\ \text { SE } & 24.31 & 10.53 & 14.42\end{array}$

24 hour Feeding Group

$\begin{array}{llrr}\text { C-4 } & 99.97 & 113.94 & 102.40 \\ \text { D-4 } & 43.44 & 138.36 & 133.00 \\ \text { E-4 } & 32.36 & 38.28 & 30.00 \\ \text { H-4 } & 60.00 & 78.88 & 91.46 \\ \text { Mean } & 58.94 \ddagger & 92.36 & 89.22 \\ \text { SE } & 14.81 & 21.77 & 21.61\end{array}$

*First meal significantly greater than intervening meals, $p<0.001$.

$\dagger$ First meal significantly greater than the last meal, $p<0.05$.

$\ddagger 8$ hour and 24 hour feeding groups are significantly different, $p<0.05$.

For this 8 hour feeding group, the average meal lasted 6 min (meal duration, MD) and meal duration was positively correlated with meal size for all monkeys (range: $r=0.78$ to 0.91 ) (Table 5). The average rate of eating was $15 \mathrm{kcal} / \mathrm{min}$, which was considerably slower than the maximal rate allowed by the pump. Rate of eating of the first meal and the
TABLE 5

CORRELATIONS BETWEEN MEAL SIZE, MEAL DURATION, PREMEAL INTERVAL AND POSTMEAL INTERVAL IN MONKEYS

\begin{tabular}{|c|c|c|c|}
\hline Monkey & $\begin{array}{c}\text { Meal Size- } \\
\text { Meal } \\
\text { Duration }\end{array}$ & $\begin{array}{c}\text { Meal Size- } \\
\text { Premeal } \\
\text { Interval }\end{array}$ & $\begin{array}{l}\text { Meal Size- } \\
\text { Postmeal } \\
\text { Interval }\end{array}$ \\
\hline \multicolumn{4}{|c|}{8 hour Feeding Schedule } \\
\hline R-3 & $0.914^{*}$ & $0.792^{*}$ & 0.154 \\
\hline S-3 & $0.829^{*}$ & $0.267 \dagger$ & $0.584^{*}$ \\
\hline W-3 & $0.782 *$ & $0.384^{*}$ & -0.07 \\
\hline$X-3$ & $0.878^{*}$ & 0.029 & 0.140 \\
\hline F-4 & $0.893^{*}$ & $0.196 \S$ & $-0.284 \dagger$ \\
\hline G-4 & $0.906^{*}$ & $0.521 \dagger$ & $0.290 \dagger$ \\
\hline
\end{tabular}

24 hour Feeding Schedule

$\begin{array}{llrl}\text { H-4 } & 0.905^{*} & -0.076 & 0.093 \\ \text { C-4 } & 0.905^{*} & -0.039 & 0.362 \ddagger \\ \text { D-4 } & 0.871^{*} & 0.093 & 0.149 \ddagger \\ \text { E-4 } & 0.905^{*} & 0.018 & 0.295^{*}\end{array}$

${ }^{*} p<0.001$.

$\dagger p<0.01$.

$\ddagger p<0.05$.

$\$ p<0.07$.

last meal did not differ from the average rate, and thus rate of feeding was not a parameter which was adjusted by these monkeys under these unperturbed conditions.

Because the feeding time of this group was restricted to the 0800 to 1600 period, the interval between the last meal and the first meal of the next day was excluded from the IMI analysis. The average IMI for the $\mathbf{8}$ hour feeding group was $91 \pm 25.3 \mathrm{~min}$ (44 to $208 \mathrm{~min}$ ). As depicted in Fig. 6, more than $25 \%$ of the IMI were less than $10 \mathrm{~min}$.

\section{Feeding Patterns During Twenty-four Hour Feeding Schedule}

When food was available 24 hours per day the monkeys took 9.4 meals (range: 5 to 16 meals) per day (Table 2). Seventy-four percent of the meals (approximately 7 meals) and $78 \%$ of the total daily caloric intake were taken during the 12-hour lights-on period. Although food was always available, there was usually a long pause between meals ranging from 4 to 12 hours during the dark phase. Most often this period of non-feeding began before midnight.

The mean MS for the 24 hour feeding group was $83 \pm 18$ $\mathrm{kcal}$ (mean range: 35 to $113 \mathrm{kcal}$ ). There was no circadian variation in the MS, light phase: $84 \mathrm{kcal}$, dark phase: $82 \mathrm{kcal}$. The size of the "last meal," defined as the meal preceding the longest IMI, did not differ significantly from the size of the first meal (the meal after the long pause during the dark phase) or from the intervening meals $(89,59$, and $92 \mathrm{kcal}$, respectively, Table 4). The distribution of meal sizes showed that $31 \%$ of the MS's were less than $30 \mathrm{kcal}$ and $6 \%$ of MS's were more than $180 \mathrm{kcal}$ (Fig. 5).

The average IMI of the 24 hour group was $158 \pm 31 \mathrm{~min}$ (range: $86 \mathrm{~min}$ to $235 \mathrm{~min}$ ). This long average IMI was caused by a significantly longer IMI between the self-imposed last meal and the first meal: $( \pm=512.3 \mathrm{~min})$. When the interval 


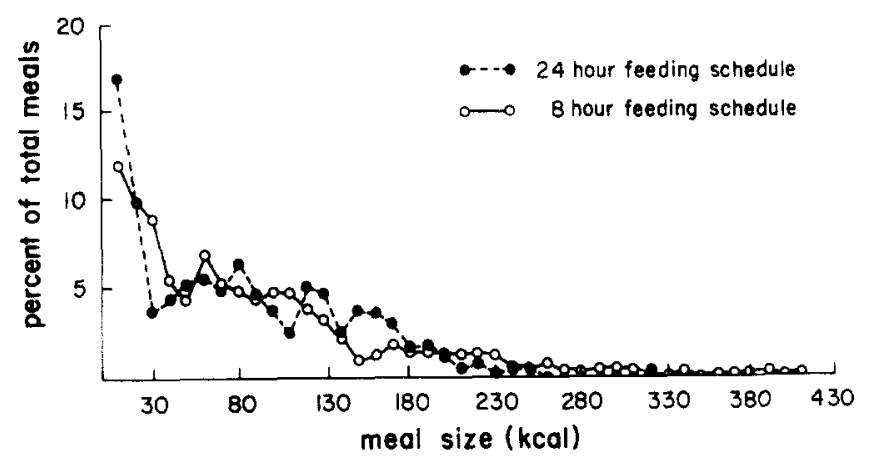

FIG. 5. Distribution of meal sizes for monkeys with 24 hours ad lib access to food compared to monkeys restricted to an 8 hours/day feeding schedule.

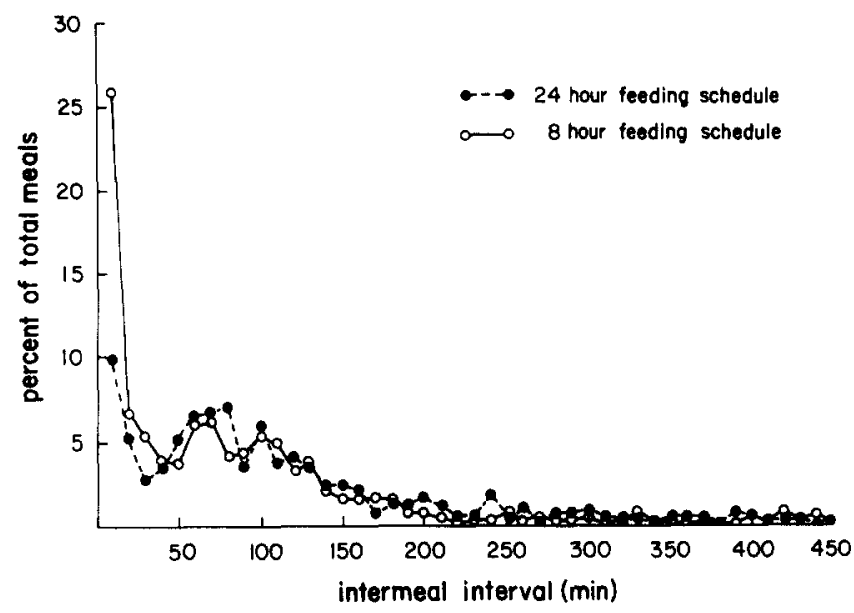

FIG. 6. Distribution of intermeal interval duration for monkeys on 24 hours and 8 hours/day feeding schedules.

after the last meal was excluded the average IMI was 121 min. The frequency distribution of the IMI's, shown in Fig. 6 , indicates the broad distribution of lengths of intervals between meals.

\section{Comparison Between 8 Hour and 24 Hour Feeding Schedules}

Monkeys in the 8 hour feeding group generally took fewer meals per day than monkeys in the 24 hour group, however, due to large variation between monkeys, this difference was not significant. The difference in MF was primarily due to the meals taken during the dark phase by the 24 hour fed monkeys.

Average size of meals did not differ between the 2 feeding schedules, although monkeys in the 8 hour group had more meals with size greater than $180 \mathrm{kcal}$. Those meals with larger size were usually the first meal of the day. The average MS of first meal was significantly greater then the mean MS of the $8 \mathrm{hr}$ feeding group $(t=2.15, p<0.05)$. In the 24 hour group, no differences were found between the first or last meal (defined on the basis of the longest IMI) and the average MS. The duration of each meal was not affected by the feeding schedule, nor was the rate of feeding.
Eight hour fed monkeys shortened their IMI s when com pared with those of the 24 hour fed monkeys. Again, due to large individual differences, no significant difference was found. Except at extremely short and very long IMI's, these two groups had similar IMI distributions (Fig. 6). The 8 hour feeding group had a higher percentage of shorter intervals and fewer long intervals when compared with those of the 24 hour feeding group.

\section{Relationship Between Meal Size and Intermed Intervals}

Raw data were used to analyze the relationship between meal size and pre- and post-meal intervals for each monkey (Table 5). Three of the 6 monkeys on the 8 hour feeding schedule showed no correlation between MS and post-meal interval, 2 showed significant positive correlation and the sixth showed significant negative correlation. Four monkeys had significant positive correlations between MS and premeal interval (range: $r=0.27$ to 0.79 ). The feeding pattern of one monkey did not show any correlation between MS and pre-meal or post-meal interval. When the first meal was excluded, one additional monkey showed a significant positive correlation between MS and post-meal interval, and another monkey showed a significant positive correlation between MS and pre-meal interval. For the 24 hour feeding schedule, none of the monkeys showed any relationship between MS and the pre-meal interval while 3 out of the 4 monkeys showed significant correlations between $\mathrm{MS}$ and post-meal interval.

In further seeking possible relationships between meal size and intermeal intervals we focused specifically on that interval which varied most widely (the longest IMI of a day) and on the meal immediately following and preceding this IMI, thus attempting to maximize the possibility of finding associations. Examining only the longest IMI for each day and the immediately preceding and following meal sizes. only one monkey showed a positive correlation between meal size and postmeal interval, and no correlations between meal size and pre-meal interval were observed. Analyzing the relationship between the size of the meal following the longest IMI of a day, and the succeeding post-meal interval again showed no correlation for any monkey

\section{DISCUSSION}

There was no significant difference in the rate of weight gain between the 8 hour and 24 hour feeding groups, between chow fed and liquid diet fed monkeys, or between caged and chair-restrained monkeys. The 10 monkeys averaged 0.133 $\mathrm{kg} /$ week weight gain which was within the normal range for the rate of weight gain for monkeys [6, 7, 23]. All monkeys gained weight gradually over an extended period, although, as found previously $[7,8]$, the rate of weight gain was not uniform per calorie of intake.

The overall feeding patterns varied widely between different monkeys, however, within monkey comparisons showed a consistency across days.

The 4 monkeys in the $24 \mathrm{hr}$ group showed a distinct circadian rhythm in feeding behavior. Seventy-eight percent of the daily intake, obtained in 7 meals, was taken during the daytime. This finding was in agreement with the observation that in their natural habitat monkeys tend to forage during the day time and rest at night. The MS did not show any rhythmic change. 
Restriction of feeding to 8 hours per day altered the feeding patterns when compared to a 24 hour/day feeding schedule. In the 8 hour feeding group the first meal of the day, and the last meal before the 16 hour fast were generally larger than the intervening meals. Monkeys on the restricted feeding schedule consumed significantly fewer calories, took slightly fewer meals and had slightly larger average MS when compared to the 24 hour feeding group. There were no schedule related differences in meal duration or eating rate. Similar reductions in total food intake, and increases in the size of the first meal were also found by Meeker and Myers [16]. Their 2 male monkeys took the same number of meals during the 8 hour feeding period, as was found in the present study ( 9 meals/day). However, based on an estimated caloric content of $4.3 \mathrm{kcal} / \mathrm{g}$ for Purina Monkey pellets, their monkeys consumed more calories per day and per meal than in the present experiment. No rate of weight gain data were reported in the Meeker and Myers study, so we cannot determine whether their monkeys were growing at a more rapid rate. There were no significant differences in the rate of weight gain between 8 hour and 24 hour feeding groups in the present study, although the 8 hour group did show slightly lower rates of weight gain and lower feeding efficiency than the 24 hour group.

Natelson and Bonbright [18] studying monkeys ingesting pellets also found caloric intakes and meal frequencies similar to those of the present experiments. Meal durations were longer in the Natelson and Bonbright experiment than those recorded in the present study probably due to the use of a dry pellet diet and intermittent drinking during feeding.

In the 24 hour feeding group, the size of the meal after the long pause during the night ( 4 to 12 hours) was not different from the size of other meals, however, in the 8 hour feeding group, the first meal of the day was significantly larger than all others.

In any examination and description of feeding behavior, the somewhat arbitrary selection of a definition of a "meal" is crucial to the nature of the data obtained and the conclusions drawn. A meal can be defined on the basis of some minimum amount of time following eating necessary to separate one meal from the next. Further, multiple combinations of minimal meal size plus minimal intermeal interval can be adopted.

Because we wished maximum sensitivity, and the ability to detect small changes in MS/IMI relationships we chose to define a meal as ingestion of no less that $2 \mathrm{ml}$ and passage of at least $2 \mathrm{~min}$ in order to define separate meals. This choice also permitted secondary analyses using other larger size and/or larger interval definitions of a meal.

The finding that over $30 \%$ of the meals recorded during these two feeding schedules contained less than $30 \mathrm{kcal}$, and that the average meal duration was 5-6 min indicates that the monkeys generally adopted a nibbling pattern. Larger meal sizes or longer intermeal intervals in meal definitions tended to obscure the nibbling pattern and failed to differentiate the characteristic patterns of monkeys habitually ingesting few large meals from those ingesting multiple small meals. We are satisfied that the $2 \mathrm{ml} / 2 \mathrm{~min}$ definition has optimized the analysis of relationships between meal size and intermeal intervals.

Natelson and Bonbright [18] defined the meal as at least 9 kcal ( 3 pellets) separated by $5 \mathrm{~min}$. However, data were reported based on a $20 \mathrm{~min}$ IMI criterion. Meeker and Myers [16] defined a meal as a minimum of $21.5 \mathrm{kcal}(5 \mathrm{~g})$ and an IMI of at least $18 \mathrm{~min}$. Although both studies adopted larger meals and longer intervals than the one used in the present study, the identification of number of meals/day were similar for all three studies.

The observation that the amount of food taken in one meal determines the interval after this meal and before the onset of the next one was first made by LeMagnen and Tallon [13]. Since then, numerous studies have been devoted to investigating this apparent feedback control of food intake. The results of these studies have not been consistent. Both positive correlations and no correlations between meal size and post-meal intervals have been reported in studies on rats $[1,2,3,4,12,20,21,22]$, cats [9], and monkeys [16,18]. One factor which has intervened in the analysis of the results and complicated comparisons has been the method used to analyze the feeding data. Ordering the raw data into deciles, a method used by some investigators $[13,20]$ produced higher MS-post-meal interval correlations than that obtained from raw data [19]. De Castro [4] argued that raw data should be used in order to avoid any statistical bias. In the present study, ungrouped raw data were used to analyze the correlations between MS and pre- and post-meal interval. Three out of 4 monkeys in the 24 hour feeding group showed significant correlations between MS and post-meal interval. No 24 hour feeding monkey showed a significant correlation between MS and pre-meal interval. However, since only a small proportion of the variance can be explained by this MS and post-meal interval correlation, other unknown factors are also likely to be involved. This finding is in agreement with the finding of Natelson and Bonbright in monkeys [18] and in other species during 24 hour ad lib feeding schedules [1, 4, $12,13,21,22]$. Meeker and Myers [16] did not find any consistent correlations for their chow fed monkeys. The lack of correlation in their experiment could be related to the operant task required in order for the monkeys to obtain food. In the Meeker and Myers experiment, monkeys performed an operant task of bar pressing on a fixed ratio schedule (FR-3) to obtain pellets. As described by Natelson and Bonbright [18], and others [10, 11, 14] imposing an operant task can significantly reduce the correlation between MS and post-meal interval.

In Natelson and Bonbright's experiments the correlation between meal size and pre-meal interval, which was not observed in the free-feeding situation, emerged as significant when only daytime feeding data were analyzed. In our 8 hour feeding group, however, 4 out of 6 monkeys showed significant correlations between MS and pre-meal interval. These data suggest that if a deprivation period of more than 12 hours is imposed in the daily feeding schedule, the length of the deprivation may serve as a hunger signal for the amount of food eaten in the next meal (MS and pre-meal interval correlation). Levitsky [14] using an operant task reported that a minimum of 4 hours IMI is required in order to get MS-pre-meal interval correlation in rats.

We conclude that (a) satiety signals resulting from the ingestion of a meal may play a more important role than hunger signals in determining the onset of the next meal in the ad lib feeding situation; (b) when forced deprivation (as in an 8 hour feeding schedule) is imposed, hunger-related signals may become more important in the regulation of food intake; (c) species differences may be important and, therefore, generalizations should be made with caution; (d) pattern of food availability ( 8 hours vs 24 hours/day) significantly alters meal parameters and the associated correlations and further emphasizes the importance of experimental constraints in the interpretation of results. 


\section{REFERENCES}

1. Balagura, S. and D. V. Coscina. Periodicity of food intake in the rat as measured by an operant response. Physiol. Behav. 3: 641-643, 1968.

2. Balagura, S. and D. V. Coscina. Influence of gastrointestinal loads on meal eating patterns. J. comp. physiol. Psychol. 69: 101-106, 1969.

3. Becker, E. E. and J. A. Grinker. Meal patterns in the genetically obese Zucker rat. Physiol. Behav. 18: 685-692, 1977.

4. DeCastro, J. M. Meal pattern correlations: facts and artifacts. Physiol. Behav. 15: 13-15, 1975.

5. Geiselman, P. J., J. R. Martin, D. A. Vanderweele and D. No vin. Multivariate analysis of meal patterning in intact and vagotomized rabbits. J. comp. physiol. Psychol. 94: 388-399, 1980.

6. Hamilton, C. L. Long term control of food intake in the monkey. Physiol. Behav. 9: 1-6, 1972.

7. Hansen, B.C. and K-L.C. Jen. Intake and body weight change in adult rhesus monkeys. In: Primates in Nutritional Research. edited by K. C. Hayes. New York: Academic Press, 1979, pp. 59.71 .

8. Hansen, B. C., K-L.C. Jen and N. Brown. Regulation of food intake and body weight in rhesus monkeys. In: The Body Weight Regulatory System: Normal and Disturbed Mechanisms, edited by L. A. Cioffi. New York: Raven Press, in press, 1980.

9. Kanarek, R. B. Availability and caloric density of the diet as determinants of meal patterns in cats. Physiol. Behav. 15: 611$618,1975$.

10. Kanarek, R. B. Energetics of meal patterns in rats. Physiol Behav. 17: 395-399, 1976.

11. Kissileff, H. R. Free feeding in normal and "recovered lateral" rats monitored by a pellet-detecting eatometer. Physiol. Behav. 5: $163-173,1970$.

12. LeMagnen, J. and M. Devos. Metabolic correlates of the meal onset in the free food intake of rats. Physiol. Behav. 5: 805-814, 1970
13. LeMagnen, J. and S. Tallon. La periodicite de la prise d aliment ad libitum du rat blanc. J. Physial., Paris 58: 323-349, 1966.

14. Levitsky, D. A. Feeding conditions and intermeal relationships Physiol. Behai. 12: 779-787, 1974.

15. McHugh, P. R, and T. H. Moran. Accuracy of the regulation of caloric ingestion in the rhesus monkey. Am. I. Physiol 235: R29-R34. 1978.

16. Meeker, R. B. and R. D. Myers. Patterns of food intake in the monkey during free or restricted feeding and in response to 2-DG or insulin injection. Phvsiol. Behav. 22: 563-574, 1979.

17. Milhaud, C. L., M. J. Klein and M. C. Merkel. A new restraining chair for rhesus monkeys (Macaca mulatta). $I$. med. Primatol. 9: 62-70, 1980.

18. Natelson, B. H. and J. C. Bonbright, Jr. Patterns of eating and drinking in monkeys when food and water are free and when they are earned. Physiol. Behav, 21: 201-213, 1978.

19. Panksepp, J. Reanalysis of feeding patterns in the rat. $J$. comp. physiol. Psychol. 82: 78-94, 1973.

20. Snowdon, C. T. Motivation, regulation and the control of meal parameters with oral and intragastric feeding. $J$. comp. physiol. Psychol. 69: 91-100, 1969.

21. Snowdon, C. T. and R. S. Wampler. Effects of lateral hypothalamic lesions and vagotomy on meal patterns in rats. $J$. comp. physiol. Psychol. 87: 399-409, 1974

22. Thomas, D. W. and J. Mayer. Meal taking and regulation of food intake by normal and hypothalamic hyperphagic rats. $J$. comp. physiol. Psvihol. 66: 643-653, 1968.

23. Van Wagnen, G. and H. R. Catchpole. Physical growth of the rhesus monkey (Macaca mulatta). Am. $J$, phys. Anthrop. 14: 245-273, 1956

24. Walike (Hansen), B. C., D. J. Campbell and R. A. Hillman. A liquid diet feeder for monkeys. J. appl. Physiol. 31: 946-947. 1971.

25. Walike (Hansen), B. C. and O. A. Smith. Regulation of food intake during intermittent and continuous cross circulation in monkeys (Macaca mulatta). J. comp. physid. Psychol. 80: 372-381, 1972. 